\title{
A Numerical Blade Element Approach to Estimating Propeller Flowfields
}

\author{
Douglas F. Hunsaker* \\ Brigham Young University, Provo, UT, 84606, USA
}

\begin{abstract}
A numerical method is presented as a low computational cost approach to modeling an induced propeller flowfield. This method uses blade element theory coupled with momentum equations to predict the axial and tangential velocities within the slipstream of the propeller, without the small angle approximation assumption common to most propeller models. The approach is of significant importance in the design of tail-sitter vertical takeoff and landing (VTOL) aircraft, where the propeller slipstream is the primary source of air flow past the wings in some flight conditions. The algorithm is presented, the model is characterized, and the results (including the results of coupling the propeller model with a lifting-line aerodynamic model) are compared with published experimental data.
\end{abstract}

\section{Nomenclature}

$B_{d} \quad=$ slipstream development factor

$b \quad=$ number of blades

$C_{l} \quad=$ section coefficient of lift

$c_{b} \quad=$ blade section chord

$D_{p} \quad=$ propeller diameter

$J \quad=$ advance ratio

$N \quad=$ number of blade sections

$R_{p} \quad=$ propeller radius

$r \quad=$ radial distance from propeller axis

$s \quad=$ normal distance to propeller plane

$V_{i} \quad=$ blade section total induced velocity

$V_{\theta i}=$ blade section induced tangential velocity

$\alpha=$ angle of attack to freestream

$\beta_{t}=$ geometric angle of attack at propeller tip

$\epsilon_{i} \quad=$ blade section induced angle of attack

$\epsilon_{\infty}=$ blade section advance angle of attack

$\Gamma \quad=$ blade section circulation

$\kappa=$ Goldstein's kappa factor

$\omega=$ propeller angular velocity

$\theta \quad=$ azimuthal angle of propeller

\section{Introduction}

TTEREST in vertical take-off and landing (VTOL) small unmanned air vehicles (SUAVs) has heightened Irecently as micro autopilots have become capable of handling flight trajectories common to VTOL missions. VTOL aircraft are much more maneuverable than fixed wing aircraft which allows them to take off autonomously without a large launch area, and provides the ability to perch and stare much like a bird. In

\footnotetext{
*Graduate Student, Mechanical Engineering, doug.hunsaker@byu.edu, AIAA Student Member.
} 
the initial design stages of such an aircraft, it is crucial to have a design tool that can model the aerodynamics of VTOL aircraft. Aerodynamic modeling of SUAV VTOL aircraft presents unique problems because VTOL aircraft experience aerodynamic forces foreign to conventional aircraft. For example, during take-off, hovering, and landing, propwash effects become dominant while freestream flow from the aircraft's forward velocity is almost negligible. Although Computational Fluid Dynamics (CFD) can and has been used as such a design tool, the time required to get results from this approach is too great to be used in the initial stages of a VTOL aircraft. Therefore, a design tool that quickly predicts the aerodynamics of a VTOL aircraft and includes propwash effects is desirable.

To facilitate the design of such a tool, the induced flowfield from a propeller must be quickly approximated. Blade element theories ${ }^{1}$ and helical vortex models ${ }^{2}$ have been employed to model propeller induced flowfields with impressive success. These propeller models have been linked with panel methods to predict the aerodynamic influence of a propeller on a wing. ${ }^{3,4}$

This paper presents a candidate numerical solution to quickly predict the induced flowfield behind a propeller. The model is a combination of various approaches taken by others. A blade element theory coupled with momentum equations is suggested similar to the approach published by Phillips. ${ }^{5}$ The slipstream geometry is modeled using a slipstream contraction factor suggested by McCormick. ${ }^{6}$ The resulting flowfield is added to the freestream velocity to create the input flowfield for the aerodynamic model. Results of the propeller model are compared with experimental data.

\section{Formulation}

\section{A. General Approach}

In order to predict the time-averaged flowfield behind a propeller, an induced velocity must be known behind the propeller. This velocity is a function of radius if the propeller axis is in line with the freestream velocity vector, and a function of radius and azimuthal angle, $\theta$, if the propeller is not aligned with the freestream. Phillips presents an approach which is not constrained to situations where the freestream velocity is aligned with the propeller axis. Thus, off-axis moments and forces from the propeller can be found. Dividing the propeller into $N$ discrete intervals, the induced velocity at each radial blade element can be found by relating the section circulation to the section induced tangential velocity as shown in Eq. (1).

$$
b \Gamma=4 \pi \kappa r V_{\theta i}
$$

Substituting Prandtl's tip loss factor ${ }^{7}$ for Goldstein's kappa factor, the following equation is produced

$$
\frac{b c_{b}}{16 r} C_{l}-\cos ^{-1}\left(\exp \left[-\frac{b\left(1-\frac{2 r}{D_{p}}\right)}{2 \sin \beta_{t}}\right]\right) \tan \epsilon_{i} \sin \left(\epsilon_{\infty}+\epsilon_{i}\right)=0
$$

which can be numerically solved for $\epsilon_{i}$. Once $\epsilon_{i}$ is known for a given blade section, the total induced velocity is found from

$$
V_{i}=\frac{\omega r \sin \epsilon_{i}}{\cos \epsilon_{\infty}}
$$

This velocity vector is then divided into its axial and tangential components.

Once the induced axial and tangential velocities are known at the propeller plane, the flowfield behind the propeller can be estimated by applying momentum equations. The slipstream radius at a distance $s$ behind the propeller is found by solving for the slipstream development factor suggested by McCormick.

$$
B_{d}=1+\frac{s}{\sqrt{s^{2}+R_{d}^{2}}}
$$

Where $B_{d}$ is the development factor and approaches 2 as the distance from the propeller plane $(s)$ ap-

proaches infinity. Using this radius, and applying conservation of mass and angular momentum as suggested by Stone, ${ }^{8}$ the development of the axial and tangential velocities throughout the slipstream are found. 


\section{B. Model Assumptions}

This approach to modeling the propeller flowfield implies a few underlying assumptions.

- The propeller affects the wing, but the wing does not affect the propeller. This allows for the combined aerodynamic and propeller models to first solve the propeller behavior and then solve for the aerodynamics of the wing in the resultant flowfield. No iterations need be performed between the flowfields of the wing and propeller, which provides for a faster solution.

- The axis of the propeller slipstream stays coincident with the axis of the propeller. This can be assumed if the forward velocity of the aircraft is always much greater than the sideslip velocity.

- There is no mixing between the slipstream and the freestream velocities. No adjustments are made at the edges of the slipstream to account for mixing with the freestream. This is obviously a faulty assumption, but accounting for these effects is beyond the scope of this initial-stage aerodynamic model.

The model used in the following results includes one additional assumption: The resultant induced velocities at any distance behind the propeller were assumed constant with varying azimuthal angle. Therfore, although the induced velocity downstream from the propeller is a function of $\theta$ when the propeller is at an angle of attack, the average velocity at that radius and distance from the propeller was taken as the induced velocity.

\section{Results}

Propellers are often advertised as being characterized by only a diameter and pitch. However, propellers include many other parameters such as chord distribution, airfoil lift and drag, pitch offset, and pitch washout. These parameters are not always included in published results. Therefore, basic assumptions were made in comparing the subsequent numerical results to experimental data and are noted where appropriate.

\section{A. Total Thrust}

Results from the model are first shown vs. experimental results published by Kotb. ${ }^{9}$ Kotb included many important parameters in his paper, which allowed for accurate propeller parameters to be used in the numerical model. However, airfoil lift and drag were assumed to match a NACA 0012 airfoil. Figure 1 displays the numerical vs. experimental values for coefficient of thrust vs. advance ratio. The numerical model appears to be slightly optimistic across the entire range of tested advance ratios. This could be due to the fact that the model makes no adjustment for mixing with the freestream at the boundaries of the propwash. The lack of modeling the mixing phenomena is apparent in subsequent plots.

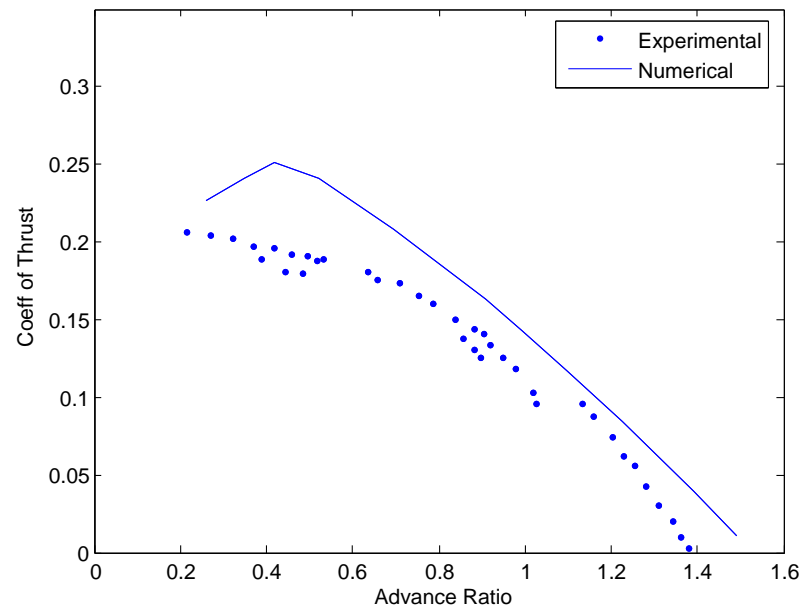

Figure 1. Experimental and numerical results for thrust coefficient vs. advance ratio. 
In situations where the propeller is an an angle of attack to the freestream, the thrust varies azimuthally around the propeller. To check the accuracy of the off-axis model, results were compared to experimental results cited by McCormick. ${ }^{6}$ Figure 2 shows how the ideal power required by the propeller varies with induced velocity and angle of attack. The velocity axis is normalized by the induced velocity under static conditions and the power axis is normalized by the power required to produce the same thrust under static conditions.

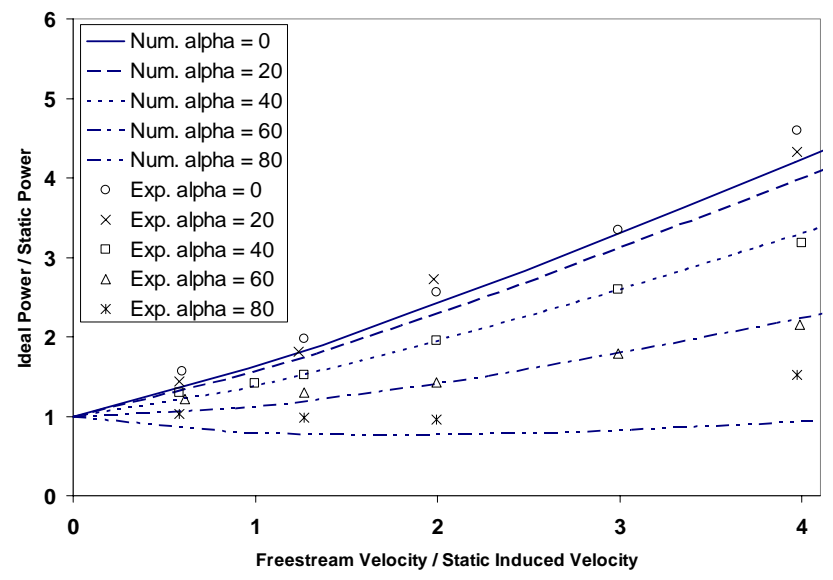

Figure 2. Required ideal power vs. velocity for constant thrust

\section{B. Velocity Profiles}

Figure 3 displays the numerical axial and tangential velocities predicted by the model vs. the time averaged experimental velocities published by Kotb. The velocities are normalized by the freestream velocity. The lack of mixing with the freestream is obvious in these plots, as the numerical results peak near the edges of the propeller, rather than tapering off into the freestream. Further discrepancies between the two sets of data could be caused by the airfoil characteristics assumption.

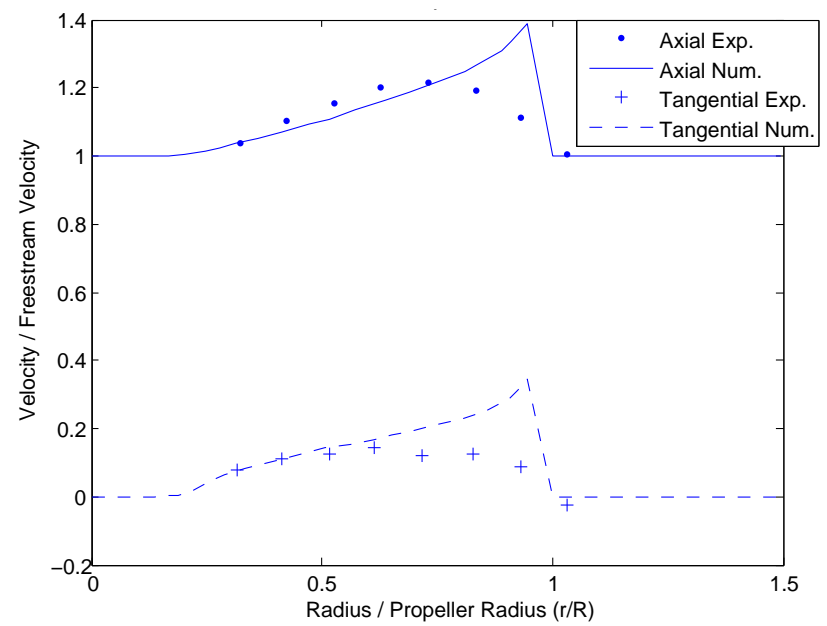

Figure 3. Normalized time-averaged velocities behind propeller vs. normalized propeller radius.

Figure 4a and Fig. 4b display the numerical results vs. the time averaged experimental data taken by Lepicovsky. ${ }^{10}$ Only basic parameters of the experimental propeller were included in the publication, so two assumptions were made: 1) the chord has an elliptical distribution, 2) The airfoil has lift and drag characteristics of a NACA 0012. From the deficit in the predicted velocity profiles in Fig. 4a, it is apparent that the propeller had an undisclosed pitch offset. By adding a pitch offset of 10 degrees, Fig. 4b was 
produced. Again, the lack of slipstream interaction with the freestream is apparent in the numerical model. However, the basic numerical velocity profiles are similar to the experimental results.

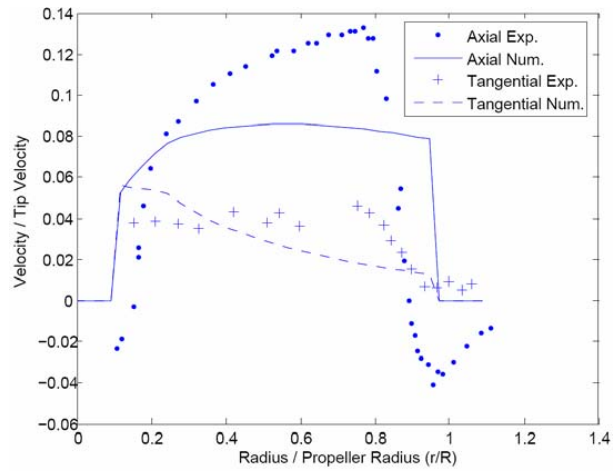

(a)

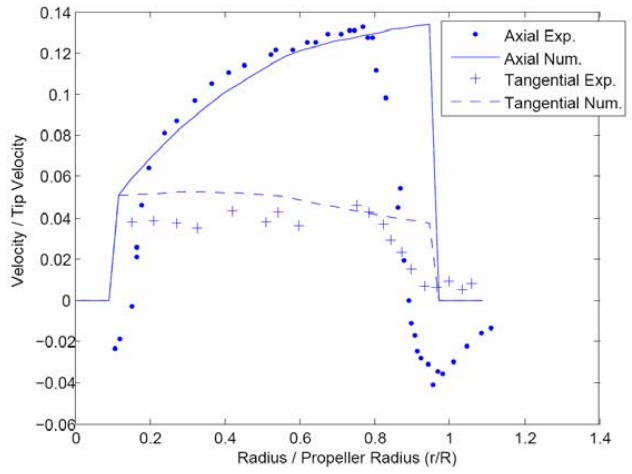

(b)

Figure 4. Normalized time-averaged velocities behind propeller vs. normalized propeller radius.

\section{Slipstream Profile}

Figure 5a displays the numerical model predictions of the slipstream radius (white line) on the experimental data taken by Nozicka. ${ }^{11}$ The data was taken at an advance ratio of 0 (static thrust condition) and reveals the strong slipstream contraction at static thrust conditions. Figure 5b displays the numerical model velocity field behind the propeller. The color coding of each of the two figures are referenced according to the color scale at the right of Fig. 5b. From the figures it can be noted that the velocity magnitudes within the slipstream of the numerical solution match experimental data quite well. The slipstream radius of the numerical model appears to become more accurate to the experimental data as it moves further downstream. Other cases were tested at various advance ratios with similar results. Again, it is apparent that no mixing with the freestream is modeled.

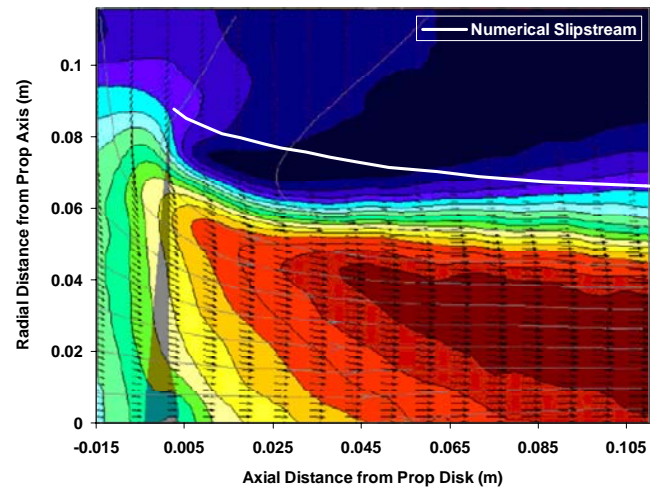

(a)

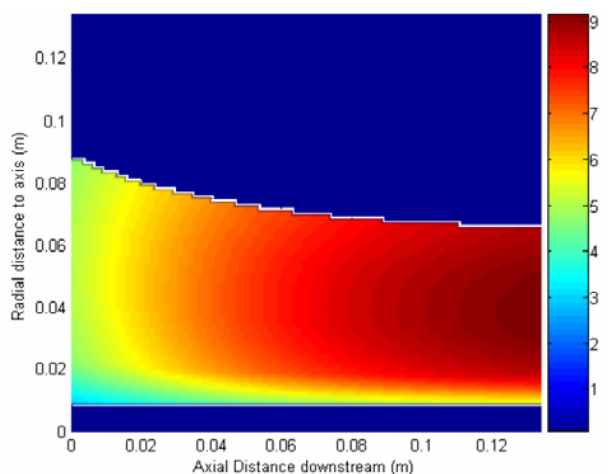

(b)

Figure 5. (a) Experimental axial velocity flowfield of a prop at $J=0$. (b) Numerical flowfield of a prop at $J$ $=0$. Color bar in units of $(\mathrm{m} / \mathrm{s})$

\section{Wing Lift Distribution}

The numerical propeller model was integrated into a numerical aerodynamic model to test how well the integrated models could predict the aerodynamics of propwash effects on an aircraft. The aerodynamic model is based off of Prandtl's lifting-line algorithm with modifications suggested by Phillips ${ }^{12}$ and further explored by the author. This approach has shown to be accurate for wings with sweep, taper, and washout and requires only a fraction of the computational time of panel methods. However, it was not known how 
well the aerodynamic model could handle rotational and non-uniform flow induced by a propeller across a wing.

A scenario was created involving the wing and propeller geometry used by Stuper ${ }^{13}$ and seen in Fig. 6 . The propeller had a diameter of $15 \mathrm{~cm}$ and a pitch of $6 \mathrm{~cm}$. The propeller was placed in the model $12.5 \mathrm{~cm}$ in front of the wing quarter chord and spun at an advance ratio of .15 in the $30 \mathrm{~m} / \mathrm{s}$ freestream. Figure 7 shows the resulting prediction for the $C_{l}$ distribution across the wing.

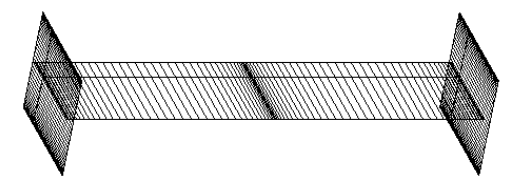

Figure 6. Computer model of finite wing showing distribution of the spanwise sections and the end-cap geometry.

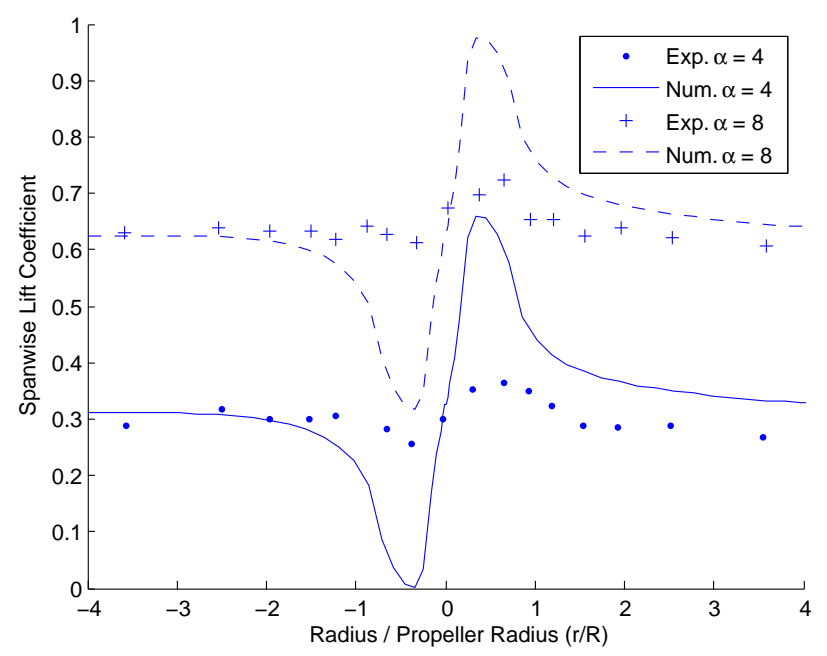

Figure 7. Numerical and experimental results for the $C_{l}$ distribution along the span of a wing in a propeller slipstream.

Note that the numerical results are qualitatively correct, but quantitatively optimistic. It should be noted, however, that little other than a standard pitch and diameter was known about the prop. It is believed that a more accurate representation of the actual propeller in the propeller model would result in better agreement with the experimental data. Additionally, the numerical lifting-line approach implemented in the aerodynamic model could be optimistically predicting the lift across the wing. Further research will include extensive analysis of this scenario as well as comparing the numerical model predictions to experimental results from Robinson ${ }^{14}$ and Brenckmann. ${ }^{15}$

\section{Conclusion}

The accuracy of the resulting propeller model is dependent upon the extent to which the propeller is correctly characterized within the model. When the propeller is correctly modeled, the propeller model predicts a slightly higher velocity profile than the experimental data. However, for the initial stages of VTOL aircraft design, the velocity profiles and slipstream geometry predicted by the model are acceptable. Thus, a method has been presented and validated which can quickly and accurately predict propeller slipstream effects on fixed wing aircraft. The speed of this solution along with its accuracy will allow the model to be used in preliminary design phases of a VTOL SUAV. 


\section{References}

${ }^{1}$ Lotstedt, P., "Propeller Slip-Stream Model in Subsonic Linearized Potential Flow," Journal of Aircraft, Vol. 29, 1992, pp. 1098-1105.

${ }^{2}$ Metcalfe, M., "On the Modelling of a Fully-Relaxed Propeller Slipstream," AIAA/SAE/ASME/ASEE 21st Joint Propulsion Conference, 1985.

${ }^{3}$ E.S. Levinsky, H.U. Thommen, P. Y. C. H., "Lifting-Surface Theory for V/STOL Aircraft in Transition and Cruise. I," Journal of Aircraft, Vol. 6, 1969, pp. 488-495.

${ }^{4}$ E.S. Levinsky, H.U. Thommen, P. Y. C. H., "Lifting-Surface Theory for V/STOL Aircraft in Transition and Cruise. II," Journal of Aircraft, Vol. 7, 1970, pp. 58-65.

${ }^{5}$ Phillips, W. F., Mechanics of Flight, John Wiley \& Sons Inc., Hoboken, New Jersey, 2004.

${ }^{6}$ McCormick, B. W., Aerodynamics of V/STOL Flight, Academic Press, New York, 1967.

${ }^{7}$ Prandtl, L. and Betz, A., Vier Abhandlungen zur Hydrodynamik und Aerodynamik, Gottingen, 1927.

${ }^{8}$ Stone, R. H., "Aerodynamic Modelling of a Wing-in-Slipstream Tail-Sitter UAV," AIAA 2002 Biennial International Powered Lift Conference and Exhibit, 2002.

${ }^{9}$ M.A. Kotb, J. S., "Measurement of Three-Dimensional Turbulent Flow Behind a Propeller in a Shear Flow," AIAA Journal, Vol. 24, 1986, pp. 570-577.

${ }^{10}$ J. Lepicovsky, W. B., "Aerodynamic Measurements About a Rotating Propeller with a Laser Velocimeter," Journal of Aircraft, Vol. 21, 1984, pp. 264-271.

${ }^{11}$ J. Noika, J. N. and Matcha, J., "Research on the Flow past an Aircraft Propeller," Proceedings of PSFVIP-4, 2003.

${ }^{12}$ Phillips, W. and Snyder, D., "Modern Adaptation of Prandtl's Classic Lifting-Line Theory," Journal of Aircraft, Vol. 37, 2000, pp. 662-670.

${ }^{13}$ Stuper, J., "Effect of Propeller Slipstream on Wing and Tail," NACA TM 874, 1938.

${ }^{14}$ Robinson, R. G. and William H. Herrnstein, J., "Wing-Nacelle-Propeller Interference for Wings of Various Spans Force and Pressure-Distributuion Tests," NACA Report No. 569, 1936.

${ }^{15}$ Brenckmann, M. E., "Experimental Investigation of the Aerodynamics of a Wing in a Slipstream," Journal of the Aeronautical Sciences, 1958. 